\title{
Melatonin Level in Primary and Secondary Fibromyalgia
}

\author{
Camelia A. Abedel-Malak ${ }^{1}$, Ali M. El-Sayed Yousef ${ }^{2}$, Sherry K. Abd El-Rahman ${ }^{3}$, \\ Hatem Mezayen ${ }^{4}$, Eman R. Amer ${ }^{5}$, Shimaa Sabry ${ }^{6}$ \\ Departments of Chemistry, Faculty of Science, Damietta University ${ }^{1,6}$, Helwan University ${ }^{4}$; \\ Internal Medicine ${ }^{2}$, Rheumatology and Rehabilitation ${ }^{3}$, Clinical Pathology ${ }^{5}$, Benha Teaching Hospital; Egypt
}

\begin{abstract}
Background: Fibromyalgia syndrome (FMS), is one of the most common causes of widespread pain and diffuse tenderness. It is characterized by reduced pain threshold (hyperallgesia) \& pain with normally innocuous stimuli (allodynia).This diffuse pain is often disease associated with wide range of other symptoms including fatigue, sleep disturbance, stiffness \& more. FMS often occur concomitantly with other rheumatologic diseases such as rheumatoid arthritis (RA), systemic lupus erthymatosus (SLE) and many other systemic connective tissue diseases. The pineal hormone melatonin (MT) exerts a variety of effects on the immune system. MT activates immune cells and enhances inflammatory cytokine and nitric oxide production. Cytokines that are strongly involved in the synovial immune and inflammatory response in rheumatoid arthritis, reach the peak concentration in the early morning, when MT serum level is at its highest concentration. Objective: The aim of this study was to assess serum melatonin level \& investigate it's correlation - if any to clinical features of fibromyalgia syndrome (FMS). Methods: This is a cross sectional case control study in which we've studied 50 FMS patients defined according to the American Colleague of Rheumatology (ACR 2010) classification criteria (Wolfe et al., 2010) ${ }^{[24]}$. They were classified into two groups: Group I: They included 25 primary FMS patients . Group II: They included 25 secondary FMS patients, they were fulfilling the classification criteria of other rheumatologic disease such as RA, SLE. Additionally, twenty age and sex matched healthy individuals were included in the study as a control group. Results: Mean Melatonin titers were significantly reduced $(\mathrm{p}<0.0001)$ in primary FMs patients compared to the controls $(21.32 \mathrm{vs} .30 .9 \mathrm{pg} / \mathrm{ml})$, but they were significantly elevated $(\mathrm{p}<0.0001)$ in secondary FMS compared to controls $(140.8 \mathrm{vs} .30 .9 \mathrm{pg} / \mathrm{ml})$. Our data imposed that, in 1ry FMS there were negative correlations of MT titers with tender points $\left(r=-0,967^{* *}, \mathrm{p}<0.0001\right)$, sleep disturbance $(\mathrm{r}=-$ 0.963**, $\left.\mathrm{p}<0.0001^{* *}\right)$, Fatigue $\left(\mathrm{r}=-0.972^{* *}, \mathrm{p}<0.001^{* *}\right)$, WPI $\left(\mathrm{r}=-0,933^{* *}, \mathrm{p}<0.0001\right)$ and SS $\left(\mathrm{r}=-0.934^{* *}, \mathrm{p}<0.0001\right)$. There were positive correlations of MT titers with cognitive symptoms $(\mathrm{r}=0.36, \mathrm{p}<0.061)$. Conclusions: In primary FMS patients melatonin level is lower than melatonin level in controls, meanwhile MT level is higher in secondary FMS patients than that of controls. There was a significant negative correlation between MT titers with tender points, sleep disturbance, fatigue, symptoms severity (SS) \& widespread pain index (WPI) in primary fibromyalgia syndrome. However, there was a positive correlation between MT level \& cognitive symptoms. [Egypt J Rheumatology \& Clinical Immunology, 2014; 2(1): 81-88]
\end{abstract}

Key Words: Fibromyalgia syndrome (FMS), Melatonin (MT), Widespread pain index (WPI) and symptom severity (SS) scale.

\section{INTRODUCTION}

Fibromyalgia syndrome (FMS) is a chronic disease with an unknown etiology, which is characterized by widespread pain in the musculoskeletal system, tender points, sleeping disorders, and chronic fatigue. Familial component, environmental factors, endocrinological factors neurotransmitter changes and psychological factors are suggested to contribute in the development of FMS $^{1}$. Most patients with fibromyalgic syndrome (FMS) complain of sleep disturbances, fatigue and pain. These symptoms might be a consequence of changed melatonin (MT) secretion, since MT is known to have sleep promoting properties.

Correspondence to Shimaa Sabry, Department of Chemistry, Faculty of Science, Damietta University; Egypt.

e-mail shimaasabry862@yahoo.com
Altered functioning of the hypothalamicpituitary-adrenal axis and altered melatonin production might modulate the sleep circadian rhythm in patients with rheumatoid arthritis.

It has been reported that patients with this syndrome have a higher serum concentration of serotonin autoantibodies not only when compared with healthy controls ${ }^{10}$, but also when compared with patients with other rheumatic diseases ${ }^{11}$. Also low serum levels of both serotonin ${ }^{22,26}$ and its precursor tryptophan ${ }^{20}$ appear to prevail in patients with FMS.

Melatonin (MT) is a very popular neurohormone frequently investigated in recent years. MT was suggested to be effective in the etiology and treatment of some diseases. MT is synthesized primarily in the pineal gland in the brain ${ }^{21}$. 


\section{PATIENTS AND METHODS}

This is a case control cross sectional observational study that included fifty FM patients, all were fulfilling the American Colleague of Rheumatology (ACR) criteria for $\mathrm{FMS}^{24}$. They were selected from the inpatient and/or the out patients' clinics of the Rheumatology and Immunology department at Benha teaching Hospital. Twenty apparently healthy subjects of comparable age and sex were included as a control group.

All patients underwent full history taking and clinical examination including assessment of FM disease activity using the 2010 ACR FMS criteria ${ }^{24}$.

A patient satisfies the diagnostic criteria for fibromyalgia if the following 3 conditions are met:

1) Widespread pain index (WPI) $>7$ and symptom severity (SS) scale score $>5$ or WPI 3-6 and SS scale score $>9$.

2) Symptoms have been present at a similar level for at least 3 months.

3) The patient does not have a disorder that would otherwise explain the pain.

Widespread Pain Index:

1) WPI: note the number areas in which the patient has had pain over the last week. In how many areas has the patient had pain Score will be between 0 and 19 .

Shoulder girdle, left; hip (buttock, trochanter), left; jaw, left; upper back; shoulder girdle, right; hip (buttock, trochanter), right; jaw, right; lower back; upper arm, left; upper leg, left; chest; neck; upper arm, right; upper leg, right; abdomen; lower arm, left; lower leg, left; lower arm, right; lower leg, right.

2) Symptoms severity (SS) scale score:

I. Fatigue, waking unrefreshed, cognitive symptoms

For the each of the 3 symptoms above, indicate the level of severity over the past week using the following scale:

$0=$ no problem

$1=$ slight or mild problems, generally mild or intermittent

$2=$ moderate, considerable problems, often present and/or at a moderate level

$3=$ severe: pervasive, continuous, lifedisturbing problems

II. Considering somatic symptoms in general, indicate whether the patient has:*

$0=$ no symptoms

$1=$ few symptoms

$2=$ a moderate number of symptoms

$3=$ a great deal of symptoms
The SS scale score is the sum of the severity of the 3 symptoms (fatigue, waking unrefreshed, cognitive symptoms) plus the extent (severity) of somatic symptoms in general. The final score is between 0 and 12 .

*Somatic symptoms that might be considered are: muscle pain, irritable bowel syndrome, fatigue/tiredness, thinking or remembering problem, muscle weakness, headache, pain/cramps in the abdomen, numbness/ tingling, dizziness, insomnia, depression etc. .

Patients were divided into:

- Group I: Twenty five (25) patients with primary fibromyalgia diagnosed according to the 2010 ACR FMS criteria .

- Group II: Twenty five (25) patients with secondary fibromyalgia diagnosed according to the 2010 ACR FMS criteria

All patients subjected to the following investigations: Rheumatoid factor (RF),

- Anti nuclear antibodies (ANA) and anti-DNA antibodies by ELISA.

- Erythrocyte sedimentation rate (ESR) using the Westergren method.

- $\quad$ C-reactive protein (CRP)

- $\quad$ Assay of Melatonin level:

Sera were collected from patients and controls and stored at $-20^{\circ} \mathrm{C}$ until use. Melatonin was quantitatively determined in the human serum by the enzyme linked immunosorbent assay (ELISA) supplied by Cusabio (Catalog No CSB-E08132h). An antibody specific to MT was bound to micro wells. Samples are then added to the micro wells with a biotin- conjugated antibody specific for Melatonin (MT) and avidin conjugated to Horseradish Peroxidase (HRP) is added to each micro wells and incubated. Then a TMB (3,3',5,5'tetramethyl-benzidine) substrate solution is added to each well. Only those wells that contain MT, biotin-conjugated antibody and enzyme-conjugated avidin will exhibit a change in color. The enzyme-substrate reaction is terminated by the addition of a sulphuric acid solution and the color change is measured spectrophotometrically at a wave lenght of $450 \mathrm{~nm} \pm 2 \mathrm{~nm}$. The concentration of melatonin is then determined in the samples.

\section{Statistical Analysis}

Statistical analysis of the results were carried out using SPSS software statistical computer package version 14.0(SPSS Inc., IL). Statistical differences between groups were determined by student's unpaired t-test. Significance was taken to be $\mathrm{p} \leq 0.05$. Correlation coefficient ( $r$ ) was used for measuring the relationship between two variables. 


\section{RESULTS}

Demographic data, clinical and laboratory features of FMS patients:

This study included 50 FMS patients; all are females $(100 \%)$ and no males $(0 \%)$, their age ranged between $17-60$ years $(30.5 \pm 9.8$ years $)$, disease duration $(5.6 \pm 1.6$ years) and MT level varying from $10-120 \mathrm{pg} / \mathrm{ml}(32.5 \pm 13.9)$.

- Group I: Included 25 patients with 1ry FMS $(50 \%)$, with a mean age of $33.2 \pm 13.8$ years, mean disease duration of $5.7 \pm 1.6$ years and mean MT level of $21.32 \pm 3.86$ (mean $\pm \mathrm{SD}$ ).

- Group II: Included 25 2ndry FMS patients $(50 \%)$, with a mean age of $31.8 \pm 12.7$ years, a mean disease duration of $5.6 \pm 1.5$ years and mean MT level of $140.8 \pm 13.16$.

- The control Group: Included 20 subjects, all are females their mean age was $41.7 \pm 15.5$ years. This group matched FMS patients as regards to age and sex.

MT level and Clinical features of FMS patients:

- In FMS patients, the titers of MT varied between 10.7 and $120.4 \mathrm{pg} / \mathrm{ml}$, with a mean of $81.06 \pm 61.6 \mathrm{pg} / \mathrm{ml}$.
- In the control group, the titers of MT varied between 18.8 and $41.4 \mathrm{pg} / \mathrm{ml}$, with a mean of $30.01 \pm 8.56 \mathrm{pg} / \mathrm{ml}$.

- There was a statistical significant difference was observed between MT level in FMS patients and the control group $(\mathrm{p}<0.011)$.

- There were a statistically significant negative correlations of between MT titers and tender points $(r=-0,967 * *, \mathrm{p}<0.0001 ;)$, sleep disturbance $(r=-0.963 * *, \mathrm{p}<0.0001 * *)$, Fatigue $(r=-0.972 * *$, $\mathrm{p}<0.001 * *)$, WPI $(r=-0,933 * *, \mathrm{p}<0.0001)$ and SS $\left(r=-0.934^{* *}, \mathrm{p}<0.0001\right)$ (Figure 1).

Additionally, there were positive correlations of MT titers with Cognitive symptoms $(r=0.36$, $\mathrm{p}<0.061)$.

- $\quad$ There were negative correlations of MT titers with tender points $(r=-0,880 * *, \mathrm{p}<0.0001$; Figure 2$)$, sleep disturbance $(r=-0.859 * *, \quad \mathrm{p}<0.0001 * *)$ (Figure 2), Fatigue $(r=-0.167 * *, \quad \mathrm{p}<0.001 * *)$ (Figure 2), WPI $(r=-0,812 * *, \mathrm{p}<0.0001)$ (Figure 2) and SS $\left(r=-0.799^{* *}, \mathrm{p}<0.0001\right)$ (Figure 2$)$.

Moreover, there were positive correlations of MT titers with Cognitive symptoms $(r=0.04$, $\mathrm{p}<0.848$ ) (Figure 2).

Table 1. Demographic data of FMS Patients and controls.

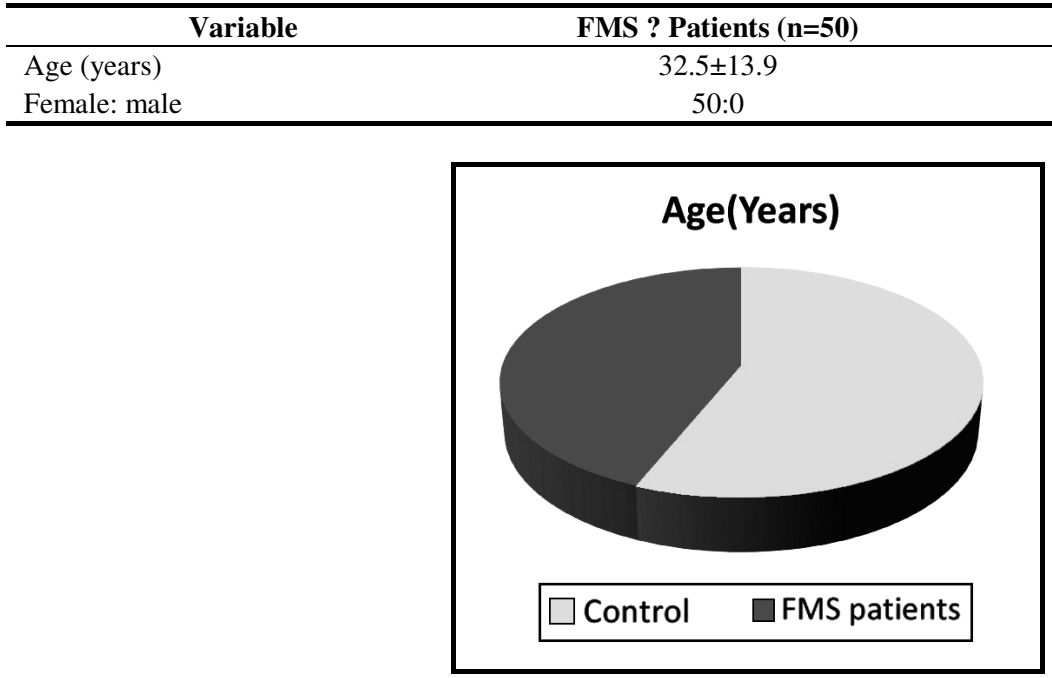

Table 2. Comparison between MT level in healthy controls and FMS patients.

\begin{tabular}{lcc}
\hline \multicolumn{1}{c}{ Group } & MT $($ mean \pm SD) & P-value \\
\hline Healthy $(\mathrm{n}=20)$ & $30.9 \pm 8.1$ & $<0.0001$ \\
1ry FMS patients $(\mathrm{n}=25)$ & $21.32 \pm 3.86$ & $<0.0001$ \\
2ry FMS patients $(\mathrm{n}=25)$ & $140.8 \pm 13.16$ & $<0.0001$ \\
FMS patients $(\mathrm{n}=50)$ & $81.06 \pm 61.1$ & $<0.011$ \\
\hline
\end{tabular}


Table 3. Correlation of MT level with clinical data of group I.

\begin{tabular}{|c|c|c|}
\hline Clinical Variable & $\mathbf{r}$ & P-value \\
\hline Tender points & $-0.967 * *$ & $<0.0001$ \\
\hline Cognitive symptoms & 0.36 & $<0.061$ \\
\hline Sleep disturbance & $-0.963 * *$ & $<0.0001$ \\
\hline Fatigue & $-0.972 * *$ & $<0.0001$ \\
\hline WPI & $-0.933 * *$ & $<0.0001$ \\
\hline SS & $-.934 * *$ & $<0.0001$ \\
\hline Age & $-0.844 * *$ & $<0.0001$ \\
\hline
\end{tabular}

** Correlation is significant at the 0.01 level (2-tailed).

Table 4. Correlation of MT level with clinical data of group II patients.

\begin{tabular}{|c|c|c|}
\hline Clinical Variable & $\mathbf{r}$ & P-value \\
\hline Tender points & $-0.880 * *$ & $<0.0001$ \\
\hline Cognitive symptoms & 0.04 & 0.848 \\
\hline Sleep disturbance & $-0.859 * *$ & $<0.0001$ \\
\hline Fatigue & $-0.167 * *$ & $<0.0001$ \\
\hline WPI & $-0.812 * *$ & $<0.0001$ \\
\hline SS & $-0.799 * *$ & $<0.0001$ \\
\hline Age & $-0.974 * *$ & $<0.0001$ \\
\hline
\end{tabular}

** Correlation is significant at the 0.01 level (2-tailed).

Table 5. Comparison between demographic data \&clinical data in healthy controls and FMS patients.

\begin{tabular}{lccc}
\hline \multicolumn{1}{c}{ Variable } & 1ry FMS patients $(\mathbf{n = 2 5})$ & 2ry FMS patients $(\mathbf{n}=25)$ & P-value \\
\hline Tender points & $11.36 \pm 1.52$ & $11.24 \pm 1.33$ & $<0.76$ \\
Cognitive symptoms & $3.84 \pm 0.46$ & $3.8 \pm 0.44$ & $<0.113$ \\
Sleep disturbance & $2.9 \pm 1.5$ & $3.16 \pm 1.6$ & $<0.660$ \\
Fatigue & $2.2 \pm 1.2$ & $3.12 \pm 0.426$ & $<0.078$ \\
WPI & $6.92 \pm 2.1$ & $7.3 \pm 1.4$ & $<0.446$ \\
SS & $7.3 \pm 1.4$ & $8.4 \pm 1.7$ & $<0.021$ \\
MT level(pg/ml) & $21.32 \pm 3.86$ & $140.8 \pm 13.16$ & $<0.0001$ \\
Age(years) & $33.2 \pm 13.8$ & $31.8 \pm 13.7$ & \\
Disease duration (years ) & $5.7 \pm 1.6$ & $5.6 \pm 1.5$ & \\
\hline
\end{tabular}

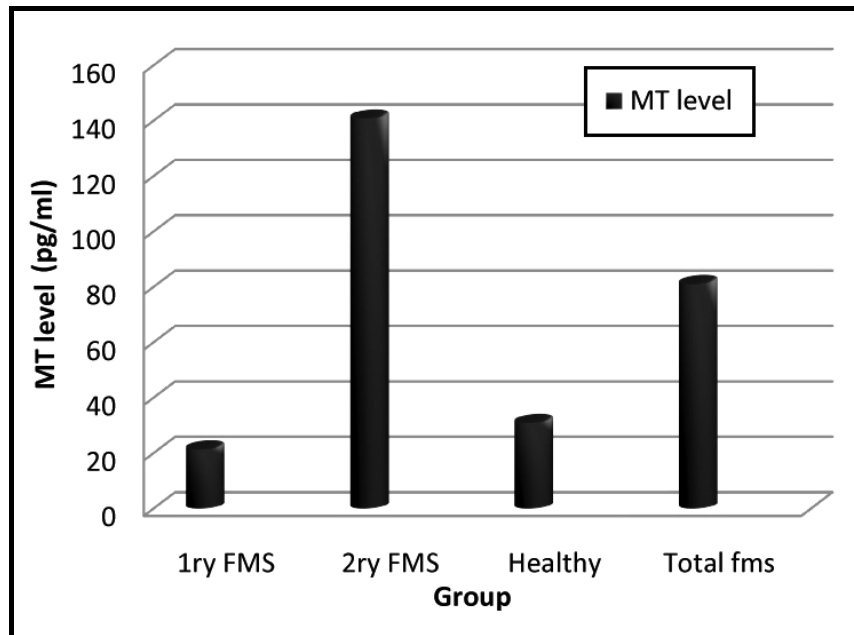

1ry FMS: primary fibromyalgia patients. 2ry FMS: secondary fibromyalgia patients.

Total FMS: total fibromyalgia patients. 

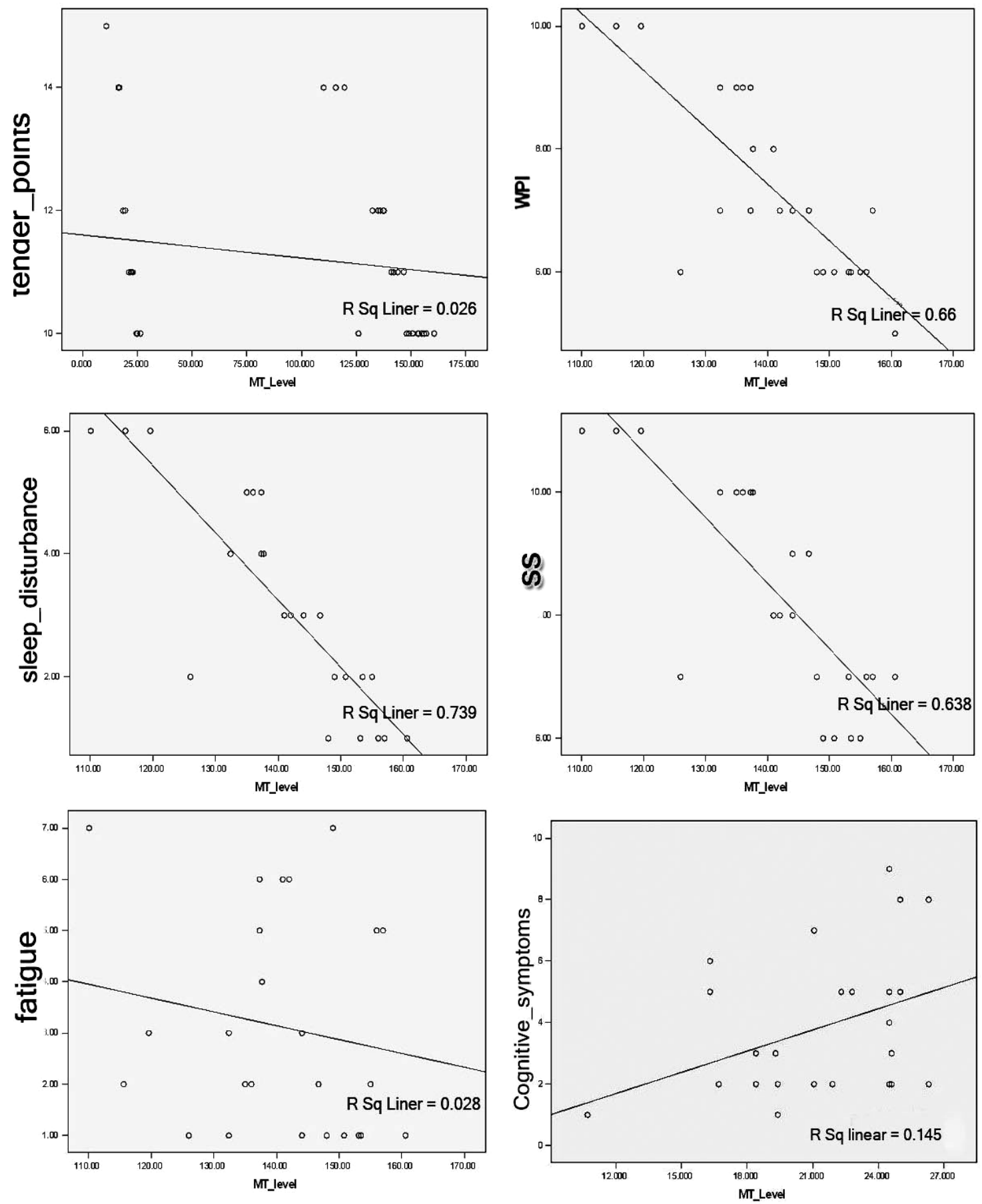

Figure 1. Negative correlation between MT and tender points, fatigue, sleep disturbance, WPI \& SS and positive correlation between MT and cognitive symptoms in primary FMS patients. 
Abedel-Malak, et al.: Melatonin level in fibromyalgia
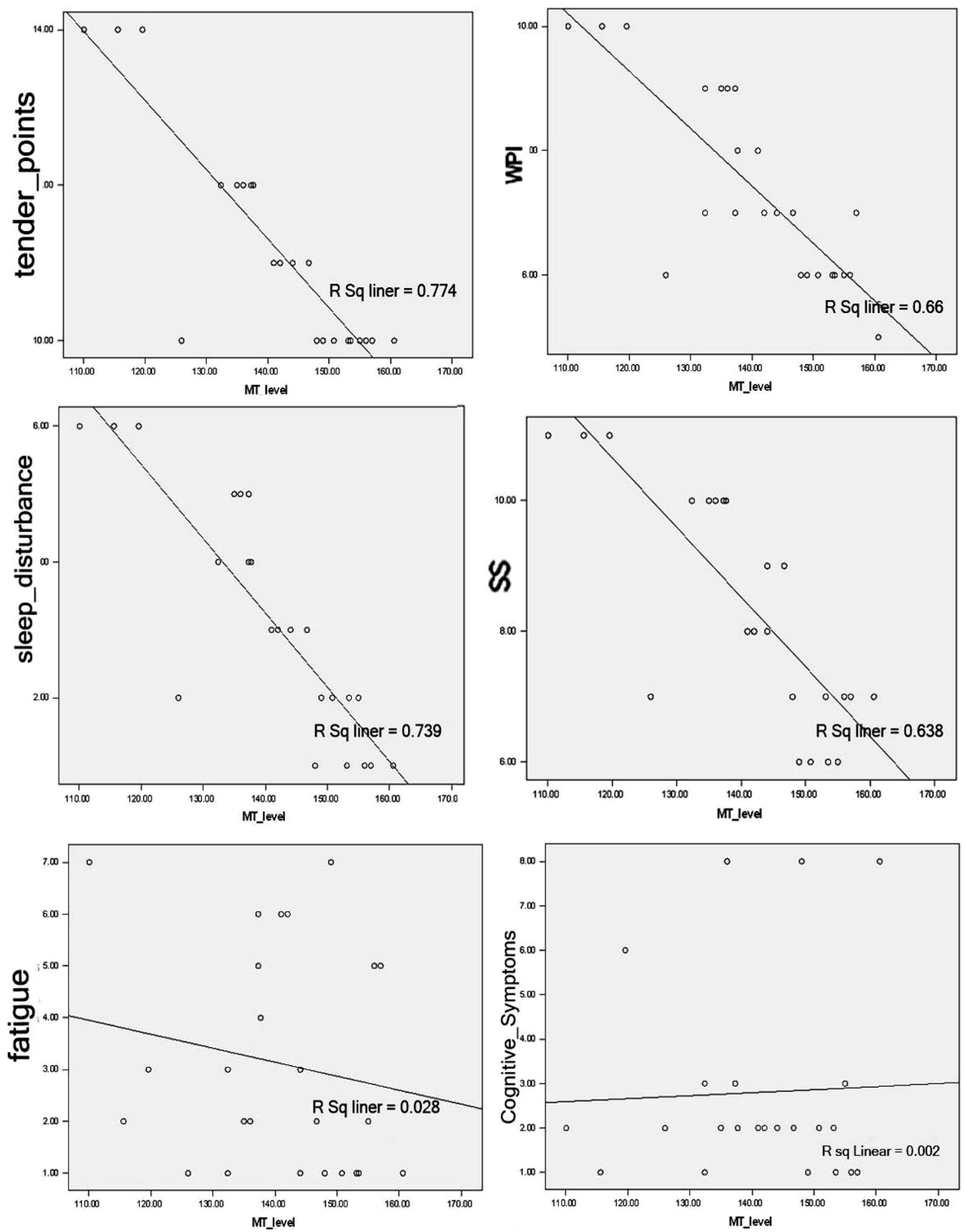

Figure 2. Negative correlation between MT and tender points, fatigue, sleep disturbance, WPI \& SS and positive correlation between MT and cognitive symptoms in secondary FMS patients. 


\section{DISCUSSION}

Fibromyalgia is a common rheumatologic disorder that is under diagnosed. Using the 1990 ACR classification criteria, the prevalence of fibromyalgia in the general population is reported to be higher than in women than in men $^{19}$.

There is a higher prevalence in females because females produce more neurotransmitters that increase pain signals and fewer neurotransmitters that decrease pain signals than males, so in our study we observe that all FM patients are females ${ }^{15}$.

Studies suggested that when endogenous tryptop han is depleted, there is a dramatic drop in synthesis of serotonin in females ${ }^{17}$. This make females being more sensitive to depression and pain (FMS) ${ }^{26}$.

The FM syndrome may coexist with other rheumatic diseases such as rheumatoid arthritis and systemic lupus erthymatosus in this case fibromyalgia is considered a secondary type ${ }^{2}$.

Disturbances in melatonin secretion have been proposed to be part of the pathophysiology of FMS.

The circadian rhythm ${ }^{12}$ of melatonin secretion have been examined in patients with $\mathrm{FMS}^{13}$, Non significant differences were observed in the 24-hr plasma melatonin level ${ }^{12}$

We found that MT level was lower in primary FMS patients compared with healthy age and sex-matched subjects. The clinical implication of this finding is disturbed sleep at night and, as consequence, daytime sleepiness, fatigue and increased pain perception. Sleep disturbances, fatigue and cognitive dysfunction, are the hall mark of FMS, present in almost all patients with $\mathrm{FMS}^{8,16}$, including those in this study.

In our study, MT levels of FMS patients were found to be significantly lower than in control group. There was positive correlation between the MT levels and pain, fatigue, and sleeping disorders. Our results weren't agree with the study of Press et al., Senel et al. \& Klerman $^{12,18,20}$.

In agreement with our results Wikner et al. ${ }^{22}$ have investigated eight patients with FM and eight controls, the concentration of melatonin in plasma and urine was found to be significantly lower in patients with FM However, Korszun et al. ${ }^{13}$ however, reported that the melatonin concentration in urine was elevated in patients with FM when compared to controls.

Our data imposed that, in 1ry FMS there were significant negative correlations of MT titers with each of tender points $(r=-0,967 * *, \mathrm{p}<0.0001)$, sleep disturbance $(r=-0.963 * *, \quad \mathrm{p}<0.0001 * *), \quad$ fatigue $(r=-0.972 * *, \quad \mathrm{p}<0.001 * *), \quad$ WPI $\quad(r=-0,933 * *$, $\mathrm{p}<0 . .0001)$ and SS $\left(r=-0.934^{* *}, \mathrm{p}<0.0001\right)$.

While, there were positive correlations of MT titers with Cognitive symptoms $(r=0.36, \mathrm{p}<0.061)$.

Some authors had reported that patients with depression have decreased secretion of MT and increased secretion of cortisol ${ }^{3}$. As most FMS patients show signs of depression, a decreased MT level may explain most of their symptoms.

Decreased MT synthesis may be brought about by impaired provision of tryptophan. The amino acid tryptophan transforms to serotonin in the pineal gland, which then transforms into MT hormone. The decrease in serotonin as a result of the decrease in the absorption of tryptophan in FMS patients was suggested to be responsible for the decrease in MT synthesis, and accordingly that this may result in the abnormal sleeping pattern and typical FMS pain ${ }^{9}$.

For patients with rheumatoid arthritis (RA), social disadvantage, psychological distress and RA severity predicted future development of $\mathrm{FMS}^{3}$.

Many studies ${ }^{5}$ confirmed that melatonin levels are increased in rheumatoid arthritis patients compared with sex and age matched healthy controls. Melatonin has been reported to stimulate the production of IL6 and Th1 related proinflammatory cytokines such as IL12, IL1\&IL2, which are involved in the pathophysiology of rheumatoid arthritis ${ }^{6}$.

Melatonin is also synthesized in human bone marrow cells ${ }^{4}$. However, it has been found to be present at a rather high concentration in synovial fluid in rheumatoid patients, and recently it has been reported that synovial macrophages from rheumatoid patients have specific binding sites for melatonin ${ }^{14}$.

Interestingly, the levels of plasma melatonin were highest in SLE patients. Plasma melatonin levels did not correlate with measures of clinical disease activity in SLE . $^{7}$

In the current study melatonin level was found to be higher in 2ry FMS patients, who are having RA/ SLE compared with healthy age and sex-matched volunteer subjects. This agrees with the studies that observed that MT levels are higher in RA/SLE patients with secondary fibromyalgias ${ }^{5}$.

Also in this study there is a significant correlation between MT level and clinical features of 2ry FMS such as sleep disturbance, fatigue $\&$ others.

Our results regarding 2ry FMS; there were a statistically significant negative correlations between MT titers with each of tender points $\left(r=-0,880^{* *}\right.$, $\mathrm{p}<0.0001)$, sleep disturbance $(\mathrm{r}=-0.859 * *, \mathrm{p}<0.0001 * *)$, fatigue $(\mathrm{r}=-0.167 * *, \mathrm{p}<0.001 * *)$, WPI $(\mathrm{r}=-0,812 * *$, $\mathrm{p}<0.0001)$ and $\mathrm{SS}\left(\mathrm{r}=-0.799^{* *}, \mathrm{p}<0.0001\right)$.

While, there were significant positive correlations of MT titers with cognitive symptoms ( $\mathrm{r}=0.04, \mathrm{p}<0.848$ ).

Melatonin level didnt seem to play a role in secondary fibromyalgia which may be induced by other factors which should be investigated in the future.

\section{Conclusion}

Our study indicated that FMS associated with sleep disturbance, fatigue \& cognitive dysfunction. Low MT level in primary fibromyalgia patients \& high MT level in secondary fibromyalgia patients was observed. There was a negative correlations between 
MT with tender points, sleep disturbance, fatigue, SS $\&$ WPI. But there was a positive correlation between MT \& cognitive symptoms.

[Disclosure: Authors report no conflict of interest]

\section{REFERENCES}

1. Acuna-Castroviejo D, Escames G, Reiter RJ (2006): Melatonin therapy in fibromyalgia. J Pineal Res 40(1): 98-99.

2. Baumgartner E, Finckh A, Cedraschi C, Vischer TL (2002): A six-year prospective study of a cohort of patients with fibromyalgia. Ann Rheum Dis; 61: 644-645.

3. Beck-Friis, J., Kjellman, B.F. \& Wetterberg, L. (1985): Serum melatonin in relation to clinical variables in patients with major depressive disorder and a hypothesis of a low melatonin syndrome. Acta Psychiatry Scandinavia, 71, 319-330.

4. Conti A, Conconi S, Hertens E, Skwarlo-Sonta K, Markowska M, Maestroni G.(2000): Evidence for MLT synthesis in mouse and human bone marrow cells. J Pineal Res;28:193-202.

5. Cutolo M, M Maestroni G J, Otsa K, Aakre O, et al., (2005):Circadian melatonin and cortisol levels in rheumatoid arthritis patients in winter time: a north and south Europe comparison Ann Rheum Dis; 64: 212-216.

6. Firestein GS (2003): Evolving concepts of rheumatoid arthritis. Nature; 423:356-61.

7. Haga H J, Brun J G, Rekvig O P (1999): Seasonal variations in activity of systemic lupus erythematosus in a subarctic region, lupus vol. 8 no. 4 269-273.

8. HäuserW, Zimmer C, Felde E, Köllner V(2008): What are the key symptoms of fibromyalgia? Results of a survey of theGerman Fibromyalgia Association [Was sind die Kernsymptome des Fibromyalgiesyndroms? Umfrageergebnisse der Deutschen Fibromyalgievereinigung.]. Schmerz; 22(2): 176-83.

9. Khaldy H, Leo'n J, Escames G, Bikjdaouene L, Garc1'a JJ, Acuna Castroviejo D (2002): Circadian rhythms of dopamine and dihydroxyphenyl acetic acid in the mouse striatum: effects of pinealectomy and of melatonin treatment. Neuroendocrinology 75(3): 201-208.

10. Klein, R. \& Berg, P.A. (1994): A comparative study on antibodies to nucleoli and 5-hydroxytryptamine in patients with fibromyalgia syndrome and tryptophaninduced eosinophilia-myalgia syndrome. Clinical Investigator, 72(7): 541-549.

11. Klein, R., Bansch, M. \& Berg, P.A. (1992): Clinical relevance of antibodies against serotonin and gangliosides in patients with primary fibromyalgia syndrome. Psychoneuroendocrinology, 17(6): 593598.

12. Klerman EB, Goldenberg DL, Brown EN, Maliszewski AM, Adler GK (2001): Circadian rhythms of women with fibromyalgia. J Clin Endocrinol Metab; 86:1034-1039.
13. Korszun A, Sackett-Lundeen L, Papadopoulos E et al(1999): Melatonin levels in women with fibromyalgia and chronic fatigue syndrome. J Rheumatol; 26: 2675-2680.

14. Maestroni G, Sulli A, Pizzorni C, Villaggio B, Cutolo M.(2002): MLT in rheumatoid arthritis: a disease promoting and modulating hormone? Clin Exp Rheumatol; 20: 872-3.

15. Mease P. Fibromyalgia syndrome (2005): review of clinical presentation, pathogenesis, outcome measures, and treatment. J Rheumatol Suppl 2005; 75: 6-21.

16. Moldofsky, H. (1982): Rheumatic pain modulation syndromes: The interrelationships between sleep, central nervous system, serotonin and pain. Advances in Neurology, 33: 51-57.

17. Nishizawa S, Benkelfat C, et al(1997): Differences between males and females in rates of serotonin synthesis in human brain. Proc National Acad Sci USA 94:530813.

18. Press J, Phillip M, Neumann L, Barak R, Segev Y, Abu-Shakra M, Buskila D (1998): Normal melatonin levels in patients with fibromyalgia syndrome. J Rheumatol 25(3): 551-555.

19. Reiter RJ, Acuna-Castroviejo D, Tan DX(2007): Melatonin therapy in fibromyalgia. Curr Pain Headache Rep 2007; 11: 339-342.

20. Senel K, Baykal T, Melikoglu MA, Erdal A, Karatay S, Karakoc A, Ugur M (2011): Serum melatonin levels in ankylosing spondylitis: correlation with disease activity. Rheumatol Int 31(1): 61-63.

21. Stratz, T., Samborski, W., Hrycaj, P., Pap, T., Mackiewicz, S., Mennet, P. \& Muller, W. (1993): Serotonin concentration in serum of patients with generalized tendomyopathy (fibromyalgia) and chronic poly- arthritis. Medizinische Klinik, 88(8): 458-462.

22. Wikner J, Hirsch U, Wetterberg L, Rojdmark S(1998): Fibromyalgia - a syndrome associated with decreased nocturnal melatonin secretion. Clin Endocrinol (Oxf); 49: 179-183.

23. Wolfe F, Häuser W, Hassett AL, Katz RS, Walitt BT(2011): The development of fibromyalgia - I: examination of rates and predictors in patients with rheumatoid arthritis (RA). Pain 2011;152(2): 291-9.

24. Wolfe F, Clauw DJ, Fitzcharles MA, Goldenberg DL, Katz RS, Mease P, et al(2010): The American College of Rheumatology preliminary diagnostic criteria for fibromyalgia and measurement of symptom severity. Arthritis and Rheumatism; 62(5): 600-10.

25. Wolfe F, Ross K, Anderson J, Russell IJ, Hebert L (1995): The prevalence and characteristics of fibromyalgia in the general population. Arthritis Rheum; 38: 19-28.

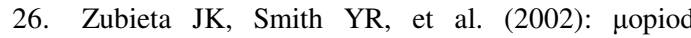
receptormediated antinociceptive responses differ in men and women. J Neurosci 22(12): 100-107. 